\title{
Current erosion indices—flawed or valid? Summary
}

\author{
Alix Young • Bennett T. Amaechi • \\ Christopher Dugmore • Peter Holbrook • June Nunn • \\ Ulrich Schiffner • Adrian Lussi • Carolina Ganss
}

Received: 29 November 2007 / Accepted: 18 December 2007 / Published online: 29 January 2008

(C) Springer-Verlag 2007

\begin{abstract}
The problem of erosive tooth wear appears increasingly to be encountered by clinicians and researchers. An adequate way of defining and recording erosive tooth wear is essential in order to assess the extent of this clinical phenomenon, both on an individual level and in the population, and for the adequate provision of preventive and therapeutic measures. Well-established erosion indices
\end{abstract}

\section{A. Young $(\bowtie)$}

Department of Cariology and Gerodontology,

Faculty of Dentistry, University of Oslo,

Oslo, Norway

e-mail: alixr@odont.uio.no

B. T. Amaechi

University of Texas Health Science Center at San Antonio,

San Antonio, TX, USA

C. Dugmore

Leicestershire County and Rutland PCT,

Leicester, UK

P. Holbrook

University of Iceland,

Reykjavik, Iceland

\section{J. Nunn}

Public and Child Dental Health, Trinity College Dublin, Dublin, Ireland

\section{U. Schiffner}

Universitätsklinikum Hamburg-Eppendorf,

Hamburg, Germany

\author{
A. Lussi \\ School of Dental Medicine, University of Bern, \\ Bern, Switzerland \\ C. Ganss \\ Dental Clinic, Justus-Liebig-University Giessen, \\ Giessen, Germany
}

have been used in most of these studies, although in many cases modifications have been made to suit the different research aims. This use of different indices is one reason why it still cannot be claimed that there is enough current knowledge on this clinical phenomenon. This article summarises the proceedings of a workshop to discuss the topic of dental erosion indices. The result of the workshop is the proposal for a new scoring system (Basic Erosive Wear Examination, BEWE) designed for use both within the research field and for dental clinicians, with the aims of standardising assessment of erosion for international comparisons, raising awareness and providing guidelines for treatment of erosive tooth wear in dental practice.

Keywords Erosive tooth wear · Index · Epidemiology · Diagnosis $\cdot$ Prevalence

\section{Introduction}

At the 1995 International Life Sciences Institute-Europe Workshop on Dental Erosion, amongst the recommendations made were the development of a clinical index for assessment of progression of dental erosion and the validation of a proposed erosion index for large-scale epidemiological surveys. One decade later, it appears that these recommendations are still relevant in that such tools have not been validated. Several different indices for the clinical diagnosis of erosive tooth wear are still in use by researchers and clinicians around the world and research on dental erosion carried out over the past decade indicates a lack of consensus. This problem applies both in relation to the prevalence, the distribution, as well as the progression of erosive tooth wear, its early detection and in the evaluation of the efficacy of preventive/therapeutic measures. Although 
both patients and dental clinicians are becoming increasingly aware of the erosive component of tooth wear, there is still a need to raise diagnostic competence through the use of a simple, standardized index that properly reflects the nature, extent and progression of the defects.

This paper summarises the result of the workshop 'Current erosion indices-flawed or valid?' held in Basle in $2007^{1}$, the aim of which was to substantiate and critically discuss the current research tools in this field.

\section{Anthropological aspects of tooth wear}

In contrast to the dental profession, anthropologists have considered tooth wear as a normal physiological phenomenon. Skeletal material from ancient remains, such as the precontemporary Australian Aboriginal populations, shows clear evidence of attrition and abrasion. The most common cause of these forms of wear is believed to be food mastication, that is to say hard, fibrous material typically consumed by these populations and tooth surface loss appears to be directly related to the age of the individual.

Anthropologists describe a tooth wear model where genetic factors influence tooth morphology and occlusion, and, in turn, occlusion and food consistency influence the chewing pattern. The resulting gradual tooth wear is accompanied by physiological adaptation involving bone remodelling and compensatory tooth eruption. It is postulated that from an evolutionary perspective, tooth wear was one of the main selective factors leading to changes in both tooth morphology and properties of dental tissues. Masticatory efficiency is strongly related to tooth anatomy and wear characteristics as highlighted in comparative studies of dentitions of many different species including herbivores, carnivores, primates and humans.

A basic understanding of the anthropological perspective of tooth wear is highly valuable both in the diagnosis and treatment planning of contemporary erosive tooth wear and particularly in relation to discussion about the relevance of erosive wear for oral health.

\section{Diagnosis and risk factors}

Dental erosion or erosive tooth wear is a multi-factorial condition. Tooth wear mechanisms seldom happen in isolation, each occurring with different intensity and duration to produce a multitude of wear patterns. This interplay is probably the reason for confusion when attempting to use any

\footnotetext{
${ }^{1}$ The workshop was held in the frame of the GABA Forum (GABA International, Münchenstein, Switzerland). We gratefully wish to thank GABA for their support.
}

tooth wear index based mainly on aetiology. Diagnosis requires a thorough knowledge of both morphological patterns typical of this type of wear and of the factors that are likely to contribute to the development of erosion.

Risk factors for erosive tooth wear can be categorized into chemical, biological and behavioural factors. The two most often cited chemical parameters, $\mathrm{pH}$ and titratable acidity, can only partly explain the erosive potential of acidic food or drinks. Mineral content, especially the common ions (calcium and phosphate) and fluoride, as well as calcium-chelation properties that vary for different dietary acids, are also important. Saliva is considered to be one important biological factor in erosion protection, but also the acquired enamel pellicle, tooth structure and positioning in relation to soft tissues and tongue may be of particular relevance. Behavioural factors can play a role in dental erosion both during and after an acidic challenge. The manner by which dietary acids are introduced and kept in the mouth before swallowing, the timing of acidic consumption/exposure and daily work/pleasure/sport activities can all have a significant effect on the development and location of erosive tooth wear.

Individuals suffering from anorexia and bulimia nervosa, rumination, chronic alcoholism or gastro-oesophageal reflux are at risk of erosive tooth wear caused by intrinsic acid and certain occupations are also conducive to erosive tooth wear such as professional wine tasting and industrial work involving exposure to acidic vapours or dust.

\section{Current tooth wear indices}

Measurement of tooth wear has been performed over the years using a large variety of both quantitative and qualitative methods. A number of indices have been developed typically using grading or scoring designed to identify increasing severity or progression of the condition, but traditionally with a focus on only one aetiological factor. Many diagnostic indices however, do not properly reflect the morphological defects and furthermore there is little international standardisation.

The earliest published attempt to diagnose tooth wear dates to the late 1800s. This index did not presuppose aetiology and formed the foundation for the development of further indices. In the latter half of the 1900s, many other indices came about largely based on work by Eccles and Jenkins [1]. The Eccles index for dental erosion of nonindustrial origin denotes three classes of lesion assigned to four tooth surfaces. A few years later, based on Eccles work, Smith and Knight [2] produced the Tooth Wear Index, a comprehensive system whereby all four visible surfaces (buccal, cervical, lingual and occlusal/incisal) of all teeth present, are scored for wear. Further modifications 
of these indices have been published, designed for specific purposes by researchers in order to suit their own work. However, some problems have been encountered concerning the amount of data produced and interand intra-examiner reliability in large-scale epidemiological surveys, as well as threshold values in different population groups. The challenge still remains to develop a simple index that can be used clinically to assess progression of wear and in epidemiological prevalence studies.

\section{Validity of current diagnostic criteria and tools for grading}

The diagnosis of tooth wear and of erosive tooth wear in particular, involves the process of identifying the condition by the lesion characteristics and by obtaining medical, nutritional, and occupational information about the patients. On an individual basis this process can be very specific compared with the case for epidemiological research where, for practical reasons, diagnosis is often restricted to lesion characteristics. Although there appears to be relatively widespread consensus as to the characteristic signs and symptoms of erosive tooth wear, dating back approximately 60 years, true validation of these clinical criteria is lacking. At that early stage, the presence of (amalgam) fillings appearing to project above the surface of the surrounding tooth was considered to be the most important clinical sign. However, hypersensitivity of the affected teeth, absence of stain and tooth wear defects with rounded margins were also mentioned. More clinically precise descriptions of the lesions have appeared in the literature over time, describing tissue loss at the gingival third of the facial surfaces that were shallow, disc-shaped, smooth, polished or with a scooped out appearance. It is currently accepted that loss of surface contour, shallow concavities on smooth surfaces, cupping and grooving on occlusal/incisal surfaces and 'proud' restorations are characteristic signs of erosive tooth wear, but reviewing the literature, it turns out that these criteria derived from observations in small groups and individual experience rather than from systematic research.

The results of epidemiological studies aimed at relating the occurrence of erosion lesions with known aetiological factors, are somewhat unclear, and in some cases controversial. Most of these studies are performed on random cross-sectional or cluster samples of children or adolescents, with few addressing the adult and aged population. Whether the current diagnostic criteria are valid in reflecting the effect of chronic acid exposure remains to be tested.

Comparative studies on wear-lesion characteristics aimed at differentiating between abrasive and erosive wear by examining dietary and nutritional differences have concluded that occlusal/incisal lesions need to be quantified by depth/breadth ratios in order to obtain a correct diagnosis, but that the presence of shallow defects on smooth surfaces is almost certainly a more valid criterion than occlusal cupping and incisal grooving. Except in cases of occlusal cupping in young individuals, it is suggested that the presence of occlusal cupping and incisal grooving are at best uncertain diagnostic criteria for erosive tooth wear, but that shallow defects on smooth surfaces coronal to the CEJ may be pathognomonic of erosive tooth wear.

Grading the degree of erosion in an individual is important as it reflects the net exposure to the erosive challenges and the opposing protective forces, and plays a part in the assessment of the problem and of the need for intervention for prevention of further tooth wear. The reliable diagnosis of both early enamel and dentine erosive tooth lesions is challenging and not very reproducible. In cases involving cupping of the molar cusp tips, determining whether dentine is exposed at the base of the cup can be very difficult. Histological studies would tend to indicate that even experienced clinicians underestimate dentine exposure.

Assessing tooth wear in the primary dentition provides further challenges. Primary teeth do not appear to withstand wear forces to the same degree as permanent teeth and commonly show signs of wear. This fact affects the scoring of severity and makes determination of aetiology in many cases more difficult.

\section{Pathological or physiological erosion: is there a relationship to age?}

In order to be able to diagnose erosive tooth wear as unacceptable or even pathological, it is imperative that acceptable levels of tooth wear be established. Partly due to the lack of effective methods for evaluating the amount of tooth loss, these data do not exist apart from a few epidemiological surveys. These studies, combined with experienced clinical judgment currently form the basis for a diagnosis of severity.

Defining pathological erosion has proved problematic. Authors addressing this concept have considered cases to be 'pathological' when excessive loss of tooth tissue has occurred affecting appearance or function, or when it causes pain. This is further complicated by attempting to predict if a tooth, at any age, would survive its current rate of wear, or, if and when interceptive treatment is required, and what level of wear is acceptable to the patient. However, an erosion lesion judged to be pathological at one age may revert to be within physiological boundaries at a later age if it remains quiescent and does not progress further during the intervening period. Thus, when defining pathology, the severity of loss, the current activity and the age of the patient must be considered. 
Furthermore, the concept of age-related pathological tooth wear becomes interesting when assessed from different perspectives. State health care authorities, private insurance companies, industry, dental clinicians and patients will all have differing interpretations of what constitutes 'pathological' tooth wear.

\section{Requirements and demands on an erosion index}

Indices have been formulated based on varying criteria, either to satisfy a particular purpose/condition or to enhance reproducibility. Among the factors considered in the development of currently existing indices are clinical versus epidemiological purposes, children versus adults, permanent versus primary teeth, full versus partial mouth scoring, exclusion/inclusion of dentine exposure and/or occlusal cupping and incisal grooving, etc. None of these indices has been found to describe dental erosion in such a way that they can be regarded as the 'gold standard' for measurement of erosion.

A simple and standardized index that is suitable for assessment of erosion should ideally be (1) easily applicable in general dental practice, (2) adaptable for epidemiological prevalence studies, (3) suitable for monitoring erosive lesion activities such as progression or arrestment of lesions, (4) easily reproducible under varying conditions for examination such as with/without magnification devices, ambient light, and hydration state of the tooth surface (dry/ wet), (5) capable of reflecting net exposure of an affected individual to the erosive challenge, (6) capable of indicating the need for treatment, and (7) should serve for both children and adult as well as permanent and primary teeth.

Development of one erosion index capable of satisfying all the above conditions poses a lot of challenges and the implications of the exclusion or inclusion of certain factors and conditions may need thorough consideration. An index involving partial scoring of the dentition used for rapid screening would be more acceptable than one incorporating full mouth scoring, which is both time consuming and believed to lower the accuracy of the index. While limiting the scoring to anterior teeth (e.g. central incisors) may eliminate a considerable number of individuals with posterior located erosion, it is considered that the use of a sextants technique, which involves a full mouth examination but scoring the most severely worn surface on each sextant, would favour easy application and reproducibility. In summary, the application of a sextant method, assessing only wear defects on the coronal smooth surfaces, and estimating severity by the diameter and depth (regardless of dentine involvement) of wear, may be considered for the development of a simple and standardised index acceptable for clinical practice and epidemiological studies.

\section{Methodological considerations}

The WHO Oral Health Program (2003) [3] focuses on oral health as an integral and essential component of general health and an important factor in quality of life. To date, only the DMFT index has been assessed and recorded on an international basis. No indicator of dental erosion is currently included in any EU or WHO oral health policy, most likely due to the lack of a standardized index for measurement of dental erosion. As part of a revision of the WHO Oral Health Surveys Basic Methods, there is now an emphasis on development of methodologies and approaches for evaluating newer disease entities such as dental erosion.

Important quality characteristics of epidemiological tools include validity and reliability and sensitivity and specificity. Content validity describes whether all aspects that are relevant for the 'construct' or content of the index are adequately considered. The content validity is based on the subjective meaning of established experts. When comparing results using a new index with results using an existing 'gold standard', it can be stated that the construct validity is high if results are well correlated with each other. When a gold standard is not available, approved hypotheses concerning the construct of interest can be confirmed empirically using the new instrument or index. Reliability of an erosion index indicates how precise that index is able to record dental erosion and encompasses both interexaminer and intra-examiner reliability. The sensitivity of an erosion index indicates its ability to detect dental erosion (compared to a gold standard) and is calculated by dividing the frequency of correct positive results by the sum of correct positive and false negative results. The specificity of an index indicates how well the index can record erosion as absent when erosion is not present and is determined by dividing the frequency of correct negative results by the sum of correct negative and false positive results.

Validation studies should be carried out on an international basis and should consider the mentioned quality criteria, most of which are relatively easy to prove. A challenge however is the question of content validity which requires research and final consensus on the construct of erosive wear.

\section{New scoring system: the Basic Erosive Wear Examination (BEWE)}

After lengthy discussions flaws in current approaches were identified and it was agreed that there is need for a validated, standardised and internationally accepted index. To initiate the development of such a tool, a universal scoring system was suggested. The new BEWE proposed in 
this issue records at the sextant level, based on the model of the BPE (Basic Periodontal Examination). It is designed to be a simple, reproducible and transferable scoring system that can be used with the diagnostic criteria of all existing indices. In time, use of the BEWE will generate comparable prevalence data, thus allowing ongoing consensus processes in the scientific community. Researchers within this field should, in practice, be able to transfer previous results using other indices based on other diagnostic criteria over to the BEWE index thereby allowing for improved comparisons.

Furthermore, the BEWE will help students, clinicians and general practitioners to screen for erosive tooth wear and to guide them in the decision-making process for the management of erosive tooth wear.

\section{Future research perspectives}

An international alliance between dentists, epidemiologists, statisticians and other scientists and politicians is recommended in order to develop an internationally agreed and accepted dental erosion index. Efforts should be made:

1. to re-evaluate existing data with the BEWE system to gain comparable data on the relevance of erosive wear for oral health and to initiate the consensus process
2. to prove the content validity of the construct 'dental erosion' and to define valid diagnostic criteria

3. to use these criteria with the BEWE system thus creating a new index

4. to validate this index on an international basis with the perspective to implement erosive wear data in the WHO and EU oral health data bases and erosion indicators in indicator sets for oral health

5. to further develop the link between the sum score of the BEWE and recommendations for prevention and treatment in the individual patient

\section{Acknowledgement}

Conflict of interest statement

The authors state that there is no conflict of interest.

\section{References}

1. Eccles JD (1979) Dental erosion of nonindustrial origin. A clinical survey and classification. J Prosthet Dent 42:649-653

2. Smith BG, Knight JK (1984) An index for measuring the wear of teeth. Br Dent J 156:435-438

3. World Health Organisation (2003) The World Oral Health Report 2003. Oral Health Programme. http://www.who.int/oral_health/ media/en/orh_report03_en.pdf 